\title{
Imaging informatics for personalised medicine: applications and challenges
}

\author{
Tianming Liu* \\ University of Georgia, \\ Athens, GA, USA \\ E-mail: tliu@uga.edu \\ ${ }^{*}$ Corresponding author

\section{Hanchuan Peng} \\ Howard Hughes Medical Institute, \\ Janelia Farm Research Campus, VA, USA
}

\section{Xiaobo Zhou}

Methodist Hospital Research Institute, Weill Medical College of Cornell University, TX, USA

\begin{abstract}
Imaging informatics has emerged as a major research theme in biomedicine in the last few decades. Currently, personalised, predictive and preventive patient care is believed to be one of the top priorities in biomedical research and practice. Imaging informatics plays a major role in biomedicine studies. This paper reviews main applications and challenges of imaging informatics in biomedicine.
\end{abstract}

Keywords: imaging; informatics; image analysis; personalised medicine.

Reference to this paper should be made as follows: Liu, T., Peng, H. and Zhou, X. (2009) 'Imaging informatics for personalised medicine: applications and challenges', Int. J. Functional Informatics and Personalised Medicine, Vol. 2, No. 2, pp.125-135.

Biographical notes: Tianming Liu is an Assistant Professor of Computer Science of the University of Georgia. Before moving to the University of Georgia, he was a faculty member of Weill Medical College of Cornell University (Assistant Professor) and Harvard Medical School (Instructor). He was a postdoc in neuroimaging in the University of Pennsylvania and Harvard Medical School. He received BS and MS in automation from Northwestern Polytechnic University, and $\mathrm{PhD}$ in computer science from Shanghai Jiaotong University. He is the recipient of the Microsoft Fellowship Award and the NIH Career Award.

Hanchuan Peng is a senior computer scientist and the head of a computational bioimage analysis lab with Janelia Farm Research Campus, Howard Hughes Medical Institute. He earned the $\mathrm{PhD}$ Degree at Southeast University, China, and did postdoctoral training at Johns Hopkins University Medical School, USA and Lawrence Berkeley National Laboratory, UC Berkeley, USA. 
$\mathrm{He}$ is a leading researcher in the field of bioimage informatics and computational biology. His recent work has been focusing on building single-neuron whole-brain level 3D digital atlases for animals including C. elegans and fruitfly. He is also known for his minimum-redundant feature selection algorithm.

Xiaobo Zhou received the MS and the PhD Degrees in Mathematics from Peking University, Beijing, China, in 1995 and 1998, respectively. He worked as a Postdoctoral Fellow in a number of institutes of Tsinghua University in Beijing, University of Missouri-Columbia in Missouri, Texas A\&M University at College Station, and Harvard Medical School at Boston. Since June 2007, he has been an Associate Professor and the Chief of Bioinformatics and Bioimaging Analysis of The Methodist Hospital Research Institute affiliated with Cornell University. His current research interests include Bioinformtaics, Bioimaging informatics, computational drug development and cancer stem cell microenvironment modelling.

\section{Introduction}

Nowadays, imaging has been playing an increasingly important role in biomedicine research towards personalised, predictive and preventive patient care. Modern biomedical imaging facilities could characterise and visualise pathological information in many human diseases at the molecular, cellular, tissue and organic scales. Effective management and interpretation of such biomedical imaging data is non-trivial. Imaging informatics becomes critical in the translation of advanced imaging techniques to the personalised patient care on the bedside, for example, development of valid imaging-based phenotypes to correlate with genotypes for personalised drug treatment. Imaging informatics mainly concerns with image acquisition, image communication, image storage and retrieval, image management, image processing and analysis, image visualisation and image interpretation. Imaging informatics already becomes a multidisciplinary research area that intersects with imaging science, medicine, computer science, information science, chemistry and engineering. In general, there are different ways to categorise imaging informatics depending on the imaging modalities used. For example, it could be classified into bioimaging informatics that mainly deals with microscopic imaging data and medical imaging informatics that mainly deals with human imaging data such as Magnetic Resonance Imaging (MRI) and CT data. This paper focuses on medical imaging informatics, and it is referred to Peng (2008) and Zhou and Wong (2008) for recent reviews on bioimaging informatics for cellular and molecular images, and the related bioinformatics applications.

The major applications of imaging informatics systems include diagnostic radiology, computer-aided diagnosis and follow-up, Image-Guided Therapy (IGT), imaging biomarker discovery and clinical trials, research-oriented knowledge discovery and personalised medicine. This paper uses nation-wide research projects as examples to demonstrate the basic concepts of imaging informatics for personalised medicine. In addition, several challenges in imaging informatics are reviewed, including integration of imaging informatics with bioinformatics and medical informatics, reliable and robust image analysis algorithms, calibration of imaging facilities, individualised disease 
mapping, data sharing and software architecture. Figure 1 provides an illustrative overview of these applications and challenges.

Figure 1 Applications and challenges of imaging informatics for personalised medicine. CADD: Computer-Aided Detection and Diagnosis. IGT: Image-guided therapy (see online version for colours)

\begin{tabular}{|l|l|l|l|}
\hline $\begin{array}{l}\text { Diagnostic } \\
\text { Radiology }\end{array}$ & CADD \\
\hline Imaging Informatics in Biomedicine
\end{tabular}

\section{Applications}

\subsection{Diagnostic radiology}

Picture Archiving and Communication Systems (PACSs) (Duerinckx and Pisa, 1982; Dwyer, 2000) is one of the earliest applications of imaging informatics system that facilitates diagnostic radiology. PACS was developed for the storage, retrieval, distribution and presentation of medical images. PACS is provided by virtually all the medical imaging equipment vendors and is adopted by virtually all hospitals. Actually, PACS imaging informatics system has revolutionised the diagnostic radiology from film-based radiology to filmless radiology (Huang, 2004).

Though PACS was originally developed for image archiving and visualisation (Dwyer, 2000), it is evolving rapidly (http://www.imagingeconomics.com/issues/ papers/MI_2008-05_01.asp). The trends of PACS development in the 21 st century are to provide clinical radiologists with automation of image processing and analysis, integration with other hospital enterprise workflows and the capability to seamlessly manage images anywhere. For example, the development of a new kind of imaging informatics system to support image-oriented clinical practice beyond the capability of current PACS for sensitive, reproducible, quantitative assessment of diseases is much needed.

\subsection{Computer-aided detection and diagnosis}

By definition, Computer-Assisted Detection and Diagnosis (CADD) is a procedure that supports and facilitates the physician's interpretations and findings in medical imaging. CADD imaging informatics system has become a major tool for early detection, early diagnosis, differential diagnosis of disease and disease staging. For example, a typical application of CADD imaging informatics system is the detection of cancer in oncological imaging, e.g., mammography screening, detection of a polyp in the colon and lung cancer detection. A variety of commercial imaging informatics systems are available for computer-aided diagnosis of breast cancer (Suri, 2006). 
In neuroimaging, informatics system has been playing an important role for differential diagnosis. For example, as definitive differential diagnosis of Alzheimer's Disease (AD) and Dementia with Lewy Bodies (DLB) requires characteristic findings at autopsy, neuroimaging poises to play an increasingly important role in research and clinical practice for differential diagnosis. Structural MRI studies (Whitwell et al., 2007) have reported that AD group with greater volume loss in the medial temporal lobe and inferior temporal regions than the DLB group, more reduction of substantia innominata grey matter in AD than DLB, and more reduction of midbrain in DLB than AD. Diffusion Tensor Imaging (DTI) studies reported significant decrease in the fractional anisotropic map in the precuneus of the DLB group than the AD group (Firbank et al., 2007).

For determination of preclinical or early stage disease, imaging is playing an increasingly greater role in the future (Silverman et al., 1999; George and Cha, 2001). Early and accurate diagnosis of AD helps patients and their families prepare for the disease and, critically, offers the best chance to treat the symptoms of the disease effectively before impairment becomes severe. Given that the neuropathological changes in $\mathrm{AD}$ are subtle and regional, accurate quantitation of such changes or abnormalities for early and differential diagnosis is a major application of imaging informatics system.

\subsection{Image-Guided Therapy}

Image-Guided Therapy aims to improve therapeutic outcomes by providing visualisation of real-time anatomical changes, navigation for target identification and intervention during intra-procedures. Enabled by IGT imaging informatics systems, surgeons and physicians can make informed decisions based on real-time visualisation and navigation. Key imaging informatics technologies in IGT include object segmentation, real-time tracking, multimodality imaging data fusion, visualisation and navigation, and software system integration.

In recent years, there have been fast-growing efforts in development of algorithms and software packages for IGT in the community (Hata et al., 2007; Cheng et al., 2006). One of the examples is the National Centre for Image-Guided Therapy (NCIGT) in Brigham and Women's Hospital (http://www.ncigt.org/). The NCIGT is an NIH-funded Biomedical Technology Resource Centre that serves as a national resource for all aspects of research related to IGT. The NCIGT provides research programmes, training and service-oriented resources for scientists and clinicians in the IGT field including image analysis and visualisation tools (Hata et al., 2007), dynamic and adaptive MRI techniques, new therapies and image-guided robotics.

\subsection{Imaging biomarker}

There has been increasing interests in the development of imaging biomarkers for disease diagnosis, disease staging and treatment monitoring. The major advantages of imaging biomarkers when compared with the clinical and cognitive measures currently in use, e.g., MMSE, are the greater reproducibility and more precise quantification. Imaging informatics plays a critical role in identifying valid imaging biomarkers from large number of measurements. For example, Alzheimer's Disease Neuroimaging Initiative (ADNI) (http://www.adni-info.org/) aims to identify valid imaging biomarkers for early diagnosis and treatment of $\mathrm{AD}$ by creating a large database of tests, measurements and 
observations taken during the progression of Mild Cognitive Impairment (MCI) and AD. This ADNI project consists of approximately 200 elderly Normal Controls (NCs), 400 subjects with MCI and approximately 200 subjects with AD, all monitored over the course of several years. Imaging informatics system was developed to archive, manage, analyse and display all MRI and PET imaging data. The raw and processed data of ADNI project is already available to ADNI investigators and the general public (http://www.loni.ucla.edu/ADNI/). Active studies are being carried on in the community to find valid imaging biomarkers that are predictive of progression of $\mathrm{AD}$.

Imaging biomarkers are actively studied in the cancer imaging community. For example, the National Institute of Cancer (NIC) has a large collaborative project of caBIG In Vivo Imaging Workspace (https://cabig.nci.nih.gov/workspaces/Imaging) that focuses on identifying how imaging informatics can be optimised and effectively integrated into the effort to relieve suffering and death from cancer. The caBIG In Vivo Imaging Workspace was launched in 2005. A large group of researchers define the needs for and participate in creating, optimising and validating methods and tools to extract meaningful information from imaging data. The tools and systems developed by the caBIG In Vivo Imaging Workspace have become the cutting-edge technologies for identifying and validating imaging biomarkers for cancer detection, diagnosis, therapy and follow-up. Many effective computational techniques to select biomarkers have been proposed (e.g., Peng et al., 2005; Ding and Peng, 2005) for minimum-redundant biomarker identification, some of them being used in pharmaceutical industry.

\subsection{Enabling research infrastructure}

The causes of many complex diseases, e.g., Schizophrenia, are largely unknown. To conduct comprehensive research on those diseases needs enabling research infrastructure that facilitates the collaboration and synergy between many researchers. One of the major applications of imaging informatics is to provide enabling research infrastructures to promote and facilitate those large-scale collaborations.

For example, the Functional Biomedical Informatics Imaging Network (fBIRN) (http://www.nbirn.net) project was to develop imaging informatics tools to make multi-site functional MRI studies a common research practice for study of Schizophrenia. The overall goals of the fBIRN project include developing an infrastructure and methodologies to conduct multi-centre fMRI studies and dissemination of these methodologies to other research groups for larger collaborative studies. Also, using Schizophrenia as a test bed, the National Alliance of Medical Image Computing (NAMIC) (http://www.na-mic.org) is a multi-institutional project that aims to develop imaging informatics methods and tools for the analysis and visualisation of medical image data. The NAMIC project combines cutting-edge computer vision research with state-of-the-art software engineering techniques to enable computational examination of the complex disease of Schizophrenia.

\subsection{Personalised medicine}

Molecular Imaging is relieved to a technology that allows physicians to diagnose, treat and monitor diseases in a personalised manner ( $\mathrm{Li}, 2002)$. For example, treating tumours with target-specific drugs has received intense attention in recent years (Storrs et al., 1995). Integration of molecular imaging into the practice of molecular medicine requires 
close collaborations among multiple scientific disciplines including biology, chemistry, imaging, clinician and engineering. Conceptually, molecular imaging can be used to facilitate target identification, to localise relevant molecular targets in vivo in a spatially and temporally, and ultimately develop personalised treatment regimens based on a combination of imaging and image-guided tissue analysis.

Imaging informatics plays important roles in translating molecular imaging into personalised medicine in the following aspects. First, imaging informatics provides the IT infrastructure and effective software tools for image archive and management, including the large amount of multimodality imaging data generated by MR, CT, PET, SPECT, High-Intensity Focused Ultrasound (HIFU) and bioluminescence/fluorescence imaging. Second, imaging informatics system supports the development and testing of novel molecular imaging agents, nanoparticles, therapeutic compounds, peptides and proteins by providing image processing and quantification tools and mathematical imaging modelling methods. Third, imaging informatics system provides visualisation tools for efficient interpretation and presentation of imaging results including multimodality imaging fusion, volume and surface rendering, interactive editing, visual tracking, visualisation of multiple data sources.

\section{Challenges}

\subsection{Integration of imaging informatics with bioinformatics and medical informatics}

In many imaging studies, there are typically other types of data sets. For example, the ADNI metadata database (http://www.loni.ucla.edu/ADNI/; http://www.adni-info.org/) consists of 63 tables, including molecular, imaging, cognitive, behavioural, clinical and demographic data. In total, the ADNI metadata dictionary contains 2547 data entries for each of the approximately 800 patients. These complex and multi-scale data points impose a tremendous challenge on data analysis and interpretation. On the one hand, it is very challenging for physicians to adequately sift through the ADNI measurements or identify what combination of them potentially could be valid biomarkers for the prediction of $\mathrm{AD}$ progression. On the other hand, pure computational algorithms may generate results that are not interpretable in clinics.

Hence, how to integrate imaging informatics tools with other bioinformatics and medical informatics tools to provide a comprehensive and integrative approach to associate imaging data with other scales of molecular and clinical data types becomes a critically important research topic in the future, as such complex and multi-scale data sets are more commonly available to answer complicated biological questions (http://www.loni.ucla.edu/ADNI/; http://www.adni-info.org/; http://www.bic.mni.mcgill. ca/nihpd/info/policies.html).

\subsection{Reliability and robustness of image analysis algorithms}

Quantitative image analysis algorithms are very important to extract mostly relevant information from imaging data. For example, segmentation of human brain structures from neuroimaging data, e.g., MRI, has been extensively studied in the past decade because of its significant importance in basic and clinical neuroscience. Segmentation of 
brain structures is of ultimate importance for definition of anatomical structures for measurement of properties of brain structures. It is very important to segment and quantify subcortical structures for better understanding of normal brain structure and function, as well as many neurological, neurodegenerative and neuropsychiatric diseases.

However, segmentation of subcortical structures is quite challenging and there are already many algorithms developed for such purpose. In general, current methods for segmentation of subcortical brain structures are in three categories: model-based methods (Shen and Davatzikos, 2000; Babalola et al., 2007; Patenaude et al., 2007), registration-based methods (Fischl et al., 1999; Qiu and Miller, 2008), hybrid methods (Corso et al., 2007; Wu and Chung, 2008). Although significant progresses in segmentation of subcortical regions have been made, current methods are still limited in their accuracy and efficiency. For example, a recent comparison study (Babalola et al., 2008) reported that, for state-of-the-art methods, the Dice Coefficients (compared with manual segmentation) are around $70 \%$ for amygdale and hippocampus, and around $80 \%$ for pallidum, caudate, putamen and thalamus. It is apparent that better approaches for subcortical structure segmentation are needed to be developed. Development of highly reliable and robust algorithm for effective and efficient quantitation of imaging parameters is of significant importance, while being very challenging.

Another example of importance of reliability and robustness of image analysis algorithms is image registration algorithm. In modern imaging research studies, it becomes essential to fuse information from multimodality imaging data, e.g., fusion of structural MRI and diffusion tensor MRI data. Development of effective and robust algorithm for co-registration of multimodality imaging data is still very challenging (Liu et al., 2004, 2006, 2007b).

\subsection{Individualised disease mapping}

Group-based statistical analysis technologies have been widely used to study the differences between diseased population and NC. Although group-based methodologies have achieved considerable success in mapping group differences, the intrinsic variability and heterogeneity in patient populations is a major obstacle for this methodology to reach the level of individualised disease mapping. For example, most of the previous results of using neuroimaging on $\mathrm{AD}$ and $\mathrm{DLB}$ were reported using statistical group analysis, meaning that they did not capture the atrophies or abnormalities at the individual patient level, though neuroanatomic areas with patient group difference were recognised (Whitwell et al., 2007; Firbank et al., 2007).

Recently, voxel-based morphometry (Ashburner and Friston, 2000) has been widely applied in the neuroimaging community to identify group difference in neuroanatomic structures, as it does not require an a priori knowledge of how brain structures are affected by disease or other factors. However, it is still a challenge to use voxel-based morphometry to infer the complex associations of features of multiple brain regions (e.g., morphology) and the respective clinical deficits (Davatzikos, 2004). Development of imaging informatics algorithms (e.g., Herskovits et al., 2004) and systems that could be able to map disease in individual patient level is of urgent needs. 


\subsection{Calibration of imaging facilities}

Many imaging studies need to integrate or associate data analysis results from multiple imaging centres to have more statistical power or to perform independent evaluation and validation. However, a couple of multi-centre neuroimaging projects have reported that imaging and data acquisition play an important role in data analysis (Friedman and Glover, 2006; Friedman et al., 2006). It is noted that such differences including MRI scanner manufacturer, RF head coil characteristics, $k$-space acquisition trajectory, reconstruction algorithms, instrumental defects and field strength differences, all contribute to the variations in results of image analysis system. For example, in fMRI studies, divergent results using ostensibly the same experimental paradigms are reported between different groups (Friedman and Birn, 2004; Friedman et al., 2004). To date, only a few studies have attempted to rationalise or correct for such differences.

The BIRN (http://www.nbirn.net) is an NIH initiative that fosters distributed collaborations in imaging science by utilising imaging informatics technology. Currently, the BIRN involves a couple of test bed projects including fBIRN and morphometry BIRN: In each of these test bed projects, there are dedicated work groups to develop novel calibration methods to allow the pooling of imaging data from a diverse demographic population as well as reduction of time necessary to accrue adequate patient populations for clinical studies of disease or therapeutic interventions using MRI.

\subsection{Data sharing}

Owing to the huge space of imaging parameter variability and tremendous heterogeneity in patient populations, pooling image data and analysis results from multiple centres is of significance to have statistically meaningful results. Hence, data sharing in imaging informatics is a fundamental issue. However, image data sharing has scientific, technical and social obstacles (Belmonte et al., 2008). For example, human imaging data sharing should meet the need for protection of patient privacy, and compliance with applicable regulations and guidelines including those of the Institutional Review Board, Health Insurance Portability and Accountability Act (HIPAA), and other institutional, local, state and federal government and community bodies.

Technically, imaging researchers would collect, store and access their data in physically different and often incompatible ways. Simply transferring data from each centre to another does not solve the problem, as the researcher-specific understanding of the image data coding and storage methods needs to be made transparent to other researchers. Effective data sharing requires a sophisticated data management system that has interoperability, scalability, transparency and integrity. The BIRN project has developed an infrastructure for imaging data sharing, however, how to adopt this infrastructure to other imaging centre is still a challenge.

\subsection{Open-architecture and open-source software system}

Software system is needed to translate imaging data and analysis result into daily decision-makings at the bedside such as computer-aided diagnosis systems, IGT systems and clinical trail systems for imaging biomarker evaluation and validation. Current software development practices demand that imaging informatics software system should have reusable modules (Liu et al., 2007a), could be scalable and sustainable to continued 
development, and interoperable with other software systems. Ideally, imaging informatics software system should be open-architecture and open-source.

There are growing efforts in the community to foster open-source and open-architecture software systems for imaging informatics. For example, the NAMIC project (http://www.na-mic.org) aims to integrate the efforts of researchers including the ITK developers (http://www.itk.org/) for development and distribution of the open-source software tools. The effort has been welcomed by the imaging informatics community. However, it takes much more effort to have a common software platform for the whole community to collaborate and exchange ideas and data.

\section{Conclusion}

The concept of personalised medicine is fast evolving. We believe all of traditional imaging modalities such as structural MRI and CT and the relatively new molecular imaging contribute to the practice of personalised medicine. Traditional imaging modalities could be used to stratify patient populations, to stage disease stages, to model disease macroenvironment, to locate diseased anatomy, to fuse multiple parameters and to monitor responses to intervention and therapy whereas molecular imaging could be used to visualise and quantify molecular and cellular events in disease states, to model molecular and cellular interactions, to guide targeted drug delivery, and to assess genotype-dependent therapies. Imaging informatics is the software platform to construct and deliver those imaging methods and tools for personalised medicine.

To conclude, in the post-genomic era, imaging informatics needs to be seamlessly integrated with bioinformatics and medical informatics to embrace the complexity of life and disease for personalised patient care.

\section{References}

Ashburner, J. and Friston, K.J. (2000) 'Voxel-based morphometry - the methods', Neuroimage, Vol. 11, No. 6, pp.805-821.

Babalola, K.O., Patenaude, B., Aljabar, P., Schnabel, J., Kennedy, D., Crum, W., Smith, S., Cootes, T.F., Jenkinson, M. and Rueckert, D. (2008) 'Comparison and evaluation of segmentation techniques for subcortical structures in brain MRI', Int. Conf. Med. Image Comput. Comput. Assist. Interv., Vol. 11, Part 1, pp.409-416.

Babalola, K.O., Petrovic, V., Cootes, T.F., Taylor, C.J., Twining, C.J., Williams, T.G. and Mills, A. (2007) 'Automated segmentation of the caudate nuclei using active appearance models. In: 3D Segmentation in the clinic: a grand challenge', Workshop Proceedings, MICCAI 2007, Brisbane, pp.57-64.

Belmonte, M.K., Mazziotta, J.C., Minshew, N.J., Evans, A.C., Courchesne, E., Dager, S.R., Bookheimer, S.Y., Aylward, E.H., Amaral, D.G., Cantor, R.M., Chugani, D.C., Dale, A.M., Davatzikos, C., Gerig, G., Herbert, M.R., Lainhart, J.E., Murphy, D.G., Piven, J., Reiss, A.L., Schultz, R.T., Zeffiro, T.A., Levi-Pearl, S., Lajonchere, C. and Colamarino, S.A. (2008) 'Offering to share: how to put heads together in autism neuroimaging', Journal of Autism and Developmental Disorders, Vol. 38, No. 1, pp.2-13.

Cheng, P., Zhang, H., Kim, H-S., Gary, K., Blake, M.B., Gobbi, D., Aylward, S., Jomier, J., Enquobahrie, A., Avila, R., Ibanez, L. and Cleary, K. (2006) 'GSTK: Framework and example application using an open source toolkit for image-guided surgery applications', Proceedings of the SPIE, Vol. 6141, pp.590-598. 
Corso, J.J., Tu, Z., Yuille, A. and Toga, A. (2007) 'Segmentation of sub-cortical structures by the graph-shifts algorithm', IPMI, Proceedings of Information Processing in Medical Imaging, pp.183-197.

Davatzikos, C. (2004) 'Why voxel-based morphometric analysis should be used with great caution when characterizing group differences', Neuroimage, Vol. 23, No. 1, pp.17-20.

Ding, C. and Peng, H. (2005) 'Minimum redundancy feature selection from microarray gene expression data', Journal of Bioinformatics and Computational Biology, Vol. 3, No. 2, pp.185-205.

Duerinckx, A.J. and Pisa, E.J. (1982) 'Filmless picture archiving and communication system (PACS) in diagnostic radiology', Proc. SPIE, Vol. 318, pp.9-18.

Firbank, M.J., Blamire, A.M., Krishnan, M.S., Teodorczuk, A., English, P., Gholkar, A., Harrison, R.M. and O'Brien, J.T. (2007) 'Diffusion tensor imaging in dementia with Lewy bodies and Alzheimer's disease', Psychiatry Res., Vol. 155, No. 2, pp.135-145.

Fischl, B., Sereno, M.I. and Dale, A.M. (1999) 'Cortical surface-based analysis II: inflation, flattening, and a surface-based coordinate system', NeuroImage, Vol. 9, pp.195-207.

Friedman, L. and Birn, F. (2004) Twelfth Annual Meeting of the ISMRM, Kyoto, Japan.

Friedman, L. and Glover, G.H. (2006) 'Report on a multicenter fMRI quality assurance protocol', Journal of Magnetic Resonance in Imaging, Vol. 23, No. 6, pp.827-839.

Friedman, L., Glover, G.H. and Birn, F. (2006) 'Reducing scanner-to-scanner variability of activation in a multi-center fMRI study: controlling for Signal-to-Fluctuation-Noise-Ratio (SFNR) differences', Neuroimage, Vol. 33, No. 2, 1 November, pp.471-481.

Friedman, L., Magnotta, V., Posse, S. and Birn, F. (2004) Twelfth Annual Meeting of the ISMRM, Kyoto, Japan.

George, A.E. and Cha, S. (2001) 'What role does functional MR imaging play in the diagnosis or prediction of future-onset Alzheimer's disease? AJNR', Am. J. Neuroradiol., Vol. 22, pp.1017-1018.

Hata, N., Piper, S., Jolesz, F.A., Tempany, C.M.C., Black, P., Morikawa, S., Iseki, H., Hashizume, M. and Kikinis, R. (2007) 'Application of open source image guided therapy software in Mr-guided therapies', Int. Conf. Med. Image Comput. Comput. Assist. Interv., Vol. 10, Part 1, pp.491-498.

Herskovits, E., Peng, H. and Davatzikos, C. (2004) 'A Bayesian morphometry algorithm', IEEE Transactions on Medical Imaging, Vol. 24, No. 6, pp.723-737.

Huang, H.K. (2004) PACS and Imaging Informatics, Wiley, ISBN 0-471-25123-2, San Francisco, CA.

Li, K.C. (2002) 'Biomedical imaging in the postgenomic era: opportunities and challenges', Acad. Radiol., Vol. 9, pp.999-1003.

Liu, T., Nie, J., Li, G., Guo, L. and Wong, S. (2007a) 'ZFIQ: a software package for zebrafish biology', Bioinformatics, Vol. 24, No. 3, pp.438-439.

Liu, T., Li, H., Wong, K., Tarokh, A., Guo, L. and Wong, S. (2007b) 'Brain tissue segmentation based on dti data', NeuroImage, Vol. 38, No. 1, pp.114-123.

Liu, T., Shen, D. and Davatzikos, C. (2004) 'Deformable registration of cortical structures via hybrid volumetric and surface warping', NeuroImage, Vol. 22, No. 4, pp.1790-1801.

Liu, T., Young, G., Huang, L., Chen, N-K. and Wong, S. (2006) '76-space analysis of grey matter diffusivity: methods and applications', Neuroimage, Vol. 15, No. 31, pp.51-65.

Patenaude, B., Smith, S., Kennedy, D. and Jenkinson, M. (2007) Bayesian Shape and Appearance Models, Technical report TR07BP1, FMRIB Centre - University of Oxford.

Peng, H. (2008) 'Bioimage informatics: a new area of engineering biology', Bioinformatics, Vol. 24, No. 17, pp.1827-1836.

Peng, H., Long, F. and Ding, C. (2005) 'Feature selection based on mutual information: criteria of max-dependency, max-relevance, and min-redundancy', IEEE Transactions on Pattern Analysis and Machine Intelligence, Vol. 27, No. 8, pp.1226-1238. 
Qiu, A. and Miller, M.I. (2008) 'Multi-structure network shape analysis via normal surface momentum maps', NeuroImage, Vol. 42, No. 4, 1 October, pp.1430-1438.

Dwyer III, S.J. (2000) 'A personalized view of the history of PACS in the USA', in Blaine, G.J. and Siegel, E.L. (Eds.): Proceedings of the SPIE, "Medical Imaging 2000: PACS Design and Evaluation: Engineering and Clinical Issues", Vol. 3980, pp.2-9.

Shen, D. and Davatzikos, C. (2000) 'An adaptive-focus deformable model using statistical and geometric information', IEEE Trans. on Pattern Analysis and Machine Intelligence (PAMI), Vol. 22, No. 8, August, pp.906-913.

Silverman, P.H.S., Chang, C.Y., Cummings, J.L. et al. (1999) 'Prognostic value of regional brain metabolism in evaluation of dementia (abstr)', J. Nucl. Med., Vol. 40, Suppl. 1, p.71.

Storrs, R.W., Tropper, F.D., Li, H.Y., Song, C.K., Kuniyoshi, J.K., Sipkins, D.A., Li, K.C.P., Bednarski, M.D. (1995) 'Paramagnetic polymerized liposome: synthesis, characterization and applications for magnetic resonance imaging', J. Am. Chem. Soc., Vol. 117, No. 28, pp.7301-7306.

Suri, J.S. (2006) Recent Advances in Breast Imaging, Mammography, and Computer-Aided Diagnosis of Breast Cancer, in Suri, J.S. and Rangayyan, R.M. (Eds.): SPIE Press Monograph Vol. PM155, 10 April.

Whitwell, J.L., Weigand, S.D., Shiung, M.M., Boeve, B.F., Ferman, T.J., Smith, G.E., Knopman, D.S., Petersen, R.C., Benarroch, E.E., Josephs, K.A. and Jack, C.R. (2007) 'Focal atrophy in dementia with Lewy bodies on MRI: a distinct pattern from Alzheimer's disease', Brain, Vol. 130, pp.708-719.

Wu, J. and Chung, A.C.S. (2008) 'Markov dependence tree-based segmentation of deep brain structures', The 11th International Conference on Medical Image Computing and Computer Assisted Intervention, MICCAI (MICCAI'08), 6-10, September, New York University, NY, USA, LNCS 5242, pp.1092-1100.

Zhou, X. and Wong, S.T.C. (2008) 'Computational systems bioinformatics and bioimaging for pathway analysis and drug screening', Proc. EEE, August, Vol. 96. No. 8, pp.1310-1331.

\section{Websites}

http://www.imagingeconomics.com/issues/articles/MI_2008-05_01.asp

http://www.ncigt.org/

http://www.loni.ucla.edu/ADNI/

https://cabig.nci.nih.gov/workspaces/Imaging

http://www.nbirn.net

http://www.na-mic.org

http://www.adni-info.org/

http://www.itk.org/

http://www.bic.mni.mcgill.ca/nihpd/info/policies.html 